International Journal of Wireless \& Mobile Networks (IJWMN) Vol. 4, No. 4, August 2012

\title{
Simplified PERFORMANCE ANALYSIS OF ENERGY DETECTORS OVER MYRIAD FADING CHANNELS: AREA UNDER THE ROC CURVE APPROACH
}

\author{
Shumon Alam, O. Olabiyi, O. Odejide, and A. Annamalai \\ Center of Excellence for Communication Systems Technology Research \\ Department of Electrical and Computer Engineering, \\ Prairie View A \& M University, TX 77446 United States of America \\ shalam2000@gmail.com, \\ engr3os@gmail.com, femiodejide@yahoo.com, aaannamalai@pvamu.edu
}

\begin{abstract}
This article investigates the energy detector's performance inmyriad fading environments by exploiting a canonical series representation of the generalized Marcum Q-function (along with higher-order derivatives of the moment generating function (MGF) of end-to-end signal-to-noise ratio (SNR) in closedform) or by a single integral formula involving the cumulative distribution function (CDF) of the end-toend SNR. The detection performance is characterized by the area under the receiver operating characteristic (ROC) curve (AUC), which is a simple statistical performance measuring metric that varies between 0.5 and 1. The proposed frameworks are capable of handling the half-odd positive integer values of the time-bandwidth products $(u)$ and the fading severity indices $(m)$ of the Nakagami-m distribution. The methods simplify the cases where many were either intractable or required complicated mathematical steps with the classical probability density function (PDF)/contour integral approaches. Several receiver diversity combining schemes are examined to demonstrate the versatility of the frameworks over independent but not necessarily identically distributed fading channels. Composite effects (multipath fading + shadowing)are also considered for the analysis. Our proposed frameworks with AUC performance metric inthe myriad fading environments provide new ways of characterizing and evaluating energy detector's performance.
\end{abstract}

\section{KEYWORDS}

Area under Curve, Diversity Combining, Energy Detector, Receiver Operating Characteristic (ROC)

\section{INTRODUCTION}

Cognitive radio (CR) holds promise to alleviate today's spectrum scarcity and underutilization problem by seeking transmission opportunities over spectral bands that are temporarily unoccupied or in a non-interfering manner and without primary user negotiation. CRs are designed to sense the environment dynamically and tune their transmission parameters rapidly to best utilize the vacant or underutilized premium spectrum real-estate[1]. Therefore, the first "cognitive" task preceding any form of dynamic spectrum management is to sense the spectrum for identification of the unoccupied white (a subband only occupied by white noise) and gray (occupied by interferers and noise) spaces. Among the various known spectrum sensing techniques, blind sensing based energy detector is perhaps the simplest (i.e., low-complexity) and most versatile since it does not require a priori knowledge of other user transmissions except of the transmission bandwidths andcenter frequencies[2].In this article, energy detector's performance is analyzed using the area under the receiver operating characteristic (ROC) curve (AUC)[3],[4]. Although, the ROC curve (probability of detection vs. probability of false alarm) or the complementary ROC curve (probability of missed detection vs. probability of false alarm)

DOI : 10.5121/ijwmn.2012.4403 
International Journal of Wireless \& Mobile Networks (IJWMN) Vol. 4, No. 4, August 2012

is capable of characterizing the energy detector's performance completely, sometimes it is difficult to compare detectors' performance using their ROC curves especially when the curves overlap.Therefore, it is desirable to have a single figure of merit that can give distinction between two detectors. Such a measure is the area under the ROC curve (AUC) which varies between 0.5 (poor performance) and 1 (good performance)[5].It is a single scalar value of expected performance and represents the probability that choosing the correct decision at the detector is more likely than choosing the incorrect decision[4]. In the worst case scenario, practical detector's performance can be described by AUC of 0.5 but the value increases to 1 as the detection improves. Even though, AUC is a useful parameter for describing detector's performance, it is only recently addressed in[5].

The research community agrees that the detection of white/gray space is more tractable by means of detection of unknown deterministic signals by energy detectors. Urkowitz [6] studied first the detection of an unknown deterministic signal over the Gaussian channel in the presence of noise through an energy detector. The presence of the energy is detected by comparing the energy of the received signal over a period with the predefined energy threshold $(\lambda)$. This method has been extended to various scenarios and diversity combining schemes. It is important to note that most detection analyses in the literature are based on the probability density function (PDF) and the analyses use the ROC curves to describe the performance ( e.g., [7]-[9]). The diversity combining isconsidered solely either over independent and identically distributed (i.i.d.) Rayleigh or Nakagami-m (restricted to positive integer fading index $m$ ) channels due to the lack of closed-form solutions for integrals involving the generalized Marcum Q-function. Moreover, the analyses are restricted to integer time-bandwidth product (TW) / sampling number $(u)$. Although Rice fading was considered in[7], the result is limited to the unity value of $u$. Contour integral is considered in[8] for correlated dual diversity for Rice channels but it is also restricted to integer $u$ value. An exponential-type contour integral is used in[9] to represent the generalized Marcum Q-function to provide a closed-form solution over i.i.d. Nakagami-m fading with positive integer $m L$ (where $L$ denotes the diversity order) for maximum ratio combining (MRC) detector. [9]also provides results for i.i.d. Rice channels using the Laurent series expansion of exponential term but the final solution is in the form of an infinite series; the summation terms require the evaluation of two separate higher order derivatives of a product term [9, eq. (27)]. Atapattu et al. [5]characterized energy detection-performance using AUC only over Nakagami-m channel and analyzed diversity combing techniques for the i.i.d. cases ( also restricted to integer $m$ and $u$ ) but the framework itself is complicated with multiple summations terms and the regularized confluent hypergeometric function of the confluent hypergeometric function ${ }_{2} F_{1}(. ; . ;$. .). Moreover, it cannot be generalized easily for other fading channels. Thus, most of the prior works have limitations; their solutions are not easy to generalize for myriad fading environments and intractable for the independent, non-identically distributed (i.n.d.) diversity combining schemes.

Thus, motivated by the current limitations, we seek simple alternative approaches for analyzing the detection-performance over myriad i.i.d./i.n.d. channel conditions.To overcome the mathematical limitations, we develop two analytical approaches to characterize the AUC performance metric over various propagation channel models (including multipath and composite effects) either by exploiting a canonical infinite series representation of the generalized Marcum Q-function (along with higher-order derivatives of the moment generating function (MGF) of end-to-end SNR in closed-form) or by a single integral formula involving the cumulative distribution function (CDF) of the end-to-end SNR. The MGF approach has advantages over other approaches and can be seen to be utilized in many recent works(see e.g.,[10]).We use the proposed frameworks subsequently to study the efficacies of diversity combining schemes such as the maximum ratio combining (MRC), square law combining (SLC), selection combining (SC) and the switch and stay combining (SSC) schemes over 
International Journal of Wireless \& Mobile Networks (IJWMN) Vol. 4, No. 4, August 2012

i.i.d./i.n.d. channels subject to multipath fading and composite effects (fading + shadowing).Furthermore, the results of this paper are more complete analysis of our initial findings that were published in[11]. The major contributions are summarized as follows.

(i) The MGF based framework that characterizes the AUC metric requires only the $k$-th derivative of the MGF of the received SNR. It is simpler than the solution given in [5]. The key advantage of this framework is that the MGF of the end-to-end SNR is readily available for the common fading conditions or it can be computed easily.

(ii) The CDF based approach requires only the CDF of the end-to-end SNR. It involves only a single integral and the computation can be done simply by numerical approach. Moreover $\mathrm{CDF}$ of the common fading environments are readily available.

(iii) The developed frameworks can be used to analyze detectors' performance in diverse propagation channel modelsand can be easily extended to various i.i.d/i.n.d. diversity combining scenarios.

(iv) The solutions are capable of handling the half-odd integer value of $u$ and $m$, which are in sharp contrast to most existing solutions.

(v) The solutions simplify the cases where many were either intractable or required complicated mathematical steps. Thus, the frameworks are simple andcomputationally efficient for analyzing detector's performance in myriad fading environments.

The rest of the paper is organized as follows. Section 2presentsthe system model for the energy detection. Section 3 includes proposed frameworks for the energy detector's performance analysis with the AUC metricfor no diversity scenarios. In Section 4, we extend the proposed frameworks for the diversity combining scenarios. Finally numerical results and concluding remarks are presented in Section 5and 6respectively.

\section{SYSTEM MODEL}

The observed waveform $y(\mathrm{t})$ of the unknown deterministic signal $s(\mathrm{t})$ at the receiver can be modeled by two hypotheses: $H_{0}$ and $H_{1}$; where hypothesis $H_{0}$ means that the signal is absent,whereas $H_{1}$ implies that the signal is present. The $y(\mathrm{t})$ can be given as[12]

$$
y(t)=\left\{\begin{array}{cc}
n(t) & : H_{0} \\
h s(t)+n(t) & : H_{1}
\end{array}\right.
$$

where, $n(\mathrm{t})$ is the additive white Gaussian noise (AWGN), $h$ is thecomplex channel gain between the primary signal transmitter and the detector. The energy detection is performed by comparing the measured energy of the observed signal in the observation time interval $T$ with the energy threshold $\lambda$. The decision statistic $Y$ can be represented as a Chi-square distribution $\chi_{2 u}^{2}$ under $H_{0}$ and a noncentralChi-square distribution $\chi_{2 u}^{2}(2 \gamma)$ with $2 u$ degree of freedom andnoncentrality parameter of $2 \gamma$ under hypothesis $H_{1}[9] . \gamma$ is the end-to-end instantaneous SNR and $u=T W$ is the time-bandwidth product ( $W$ is the filter bandwidth). Therefore, PDF of random variable $Y$ under the two hypotheses can be written as 
International Journal of Wireless \& Mobile Networks (IJWMN) Vol. 4, No. 4, August 2012

$$
f_{Y}(y)= \begin{cases}\frac{y^{u-1} e^{-y / 2}}{2^{u} \Gamma(u)}, & H_{0} \\ \frac{1}{2}\left(\frac{y}{2 u \gamma_{i}}\right)^{(u-1) / 2} e^{-(y+2 u \gamma) / 2} I_{u-1}(\sqrt{2 u \gamma y}), & H_{1}\end{cases}
$$

where, $\Gamma(\cdot)$ is the gamma function and $I_{u-1}(\cdot)$ is the modified Bessel function of the first kind. Thus, the probability of the false alarm $P_{f}=\operatorname{Pr} o b\left\{Y>\lambda \mid H_{0}\right\}$ and the probability of the detection $P_{d}=\operatorname{Pr} o b\left\{Y>\lambda \mid H_{1}\right\}$ can be derived respectively by integrating the $\operatorname{PDF}$ (for $H_{0}$ and $H_{1}$ Hypotheses) with limit $\lambda$ to $\infty$ as[13]

$$
\begin{gathered}
P_{f}(\lambda)=\Gamma\left(u, \frac{\lambda}{2}\right) / \Gamma(u) \\
P_{d}(\gamma, \lambda)=Q_{u}(\sqrt{2 \gamma}, \sqrt{\lambda})
\end{gathered}
$$

where, $Q_{u}(.,$.$) is the generalized ( u$-thorder) Marcum Q-function and $\Gamma(\cdot$,$) is the upper$ incomplete Gamma function.

\section{Energy Detection over Myriad Fading Channel Models: AUC APPROACH}

In this Section, we develop the proposed frameworks considering the AUC performance metric. We first derive the MGF based framework and then derive the CDF based simplified frameworks.

\section{1 MGF Based Framework}

AUC varies from 0.5 to 1 as the energy detector threshold $\lambda$ varies from $\infty$ to 0 [5].For a $P_{d}(\gamma, \lambda)$ vs. $P_{f}(\lambda)$ - ROC curve, instantaneous AUC can be expressed in term of instantaneous SNR $\gamma$ as

$$
A(\gamma)=\int_{0}^{1} P_{d}(\gamma, \lambda) d P_{f}(\lambda)
$$

Since $P_{f}(\lambda)$ and $P_{d}(\gamma, \lambda)$ are both functions of the threshold $\lambda$, the threshold averaging method[14] can be used in evaluating AUC. When $P_{f}(\lambda)$ varies from $0 \rightarrow 1, \lambda$ can be seen to vary from $\infty \rightarrow 0$. Thus (5) can be re-written as

$$
A(\gamma)=-\int_{0}^{\infty} P_{d}(\gamma, \lambda) \frac{\partial P_{f}(\lambda)}{\partial \lambda} d \lambda .
$$

Taking the derivative of (3) and inserting into (6) we obtain

$$
A(\gamma)=\frac{1}{2^{u} \Gamma(u)} \int_{0}^{\infty} \lambda^{u-1} e^{-\frac{\lambda}{2}} Q_{u}(\sqrt{2 \gamma}, \sqrt{\lambda}) d \lambda
$$


International Journal of Wireless \& Mobile Networks (IJWMN) Vol. 4, No. 4, August 2012

The detection probability (conditional probability) over the AWGN can be written using the canonical series representation of the generalized Marcum-Q function $Q_{u}(.,).[15]$ as (8).

$$
P_{d}(\gamma, \lambda)=Q_{u}(\sqrt{2 \gamma}, \sqrt{\lambda})=\sum_{k=0}^{\infty} \frac{\gamma^{k} e^{-\gamma}}{k !} \frac{\Gamma\left(u+k, \frac{\lambda}{2}\right)}{\Gamma(u+k)}
$$

An alternative canonical series representation of $Q_{u}(.,$.$) can be derived by substituting$ $G(a, z)=\Gamma(a)-\Gamma(a, z)$ in $(8)$, as

$$
P_{d}(\gamma, \lambda)=Q_{u}(\sqrt{2 \gamma}, \sqrt{\lambda})=1-\sum_{k=0}^{\infty} \frac{\gamma^{k} e^{-\gamma}}{k !} \frac{G\left(u+k, \frac{\lambda}{2}\right)}{\Gamma(u+k)}
$$

where, $G(a, z)$ is the lower incomplete Gamma function. Itcan be noted here that the alternative Marcum Q-functionis capable of handling the half-odd positive integer $u$ and $m$ [15].Thus (8) or (9) also hold this property congenially. Later we show that both (8) and (9) are in desirable forms that facilitate the fading averaging problem in a unified manner using the MGF-derivative approach. Now substituting (8) or (9) into (7) we can obtain the unfaded AUCrespectively as

$$
\begin{gathered}
A(\gamma)=\frac{1}{2^{u} \Gamma(u)} \sum_{k=0}^{\infty} \frac{\gamma^{k} e^{-\gamma}}{k ! \Gamma(u+k)} \frac{\Gamma(k+2 u)_{2} F_{1}(1, k+2 u ; 1+u ; 0.5)}{u 2^{(u+k)}} \\
A(\gamma)=1-\frac{1}{\Gamma(u)} \sum_{k=0}^{\infty} \frac{\Gamma(k+2 u)_{2} F_{1}(1, k+2 u ; 1+u+k ; 0.5)}{(u+k) \Gamma(k+u) k ! 2^{(2 u+k)}}
\end{gathered}
$$

where, ${ }_{2} F_{1}(., . ; ; ;$.$) is the confluent hypergeometric function. The average \operatorname{AUC}(\bar{A})$ over fading channels can be evaluated as

$$
\bar{A}=\int_{0}^{\infty} A(\gamma) f_{\gamma}(\gamma) d \gamma
$$

Substituting (10) into (12) yields average AUC as

$$
\bar{A}=\frac{1}{u 2^{u} \Gamma(u)} \sum_{k=0}^{\infty} \frac{\Gamma(k+2 u)_{2} F_{1}(1, k+2 u ; 1+u ; 0.5)}{\Gamma(k+u) k ! 2^{(u+k)}} \int_{0}^{\infty} \gamma^{k} e^{-\gamma} f_{\gamma}(\gamma) d \gamma
$$

where,

$$
\int_{0}^{\infty} \gamma^{k} e^{-\gamma} f_{\gamma}(\gamma) d \gamma=\left.(-1)^{k} \phi_{\gamma}^{(k)}(s)\right|_{s=1}
$$

is the Laplace-transform identity. $\phi_{\gamma}^{(k)}(s)$ is the $k$-th derivative of the MGF $\phi_{\gamma}(s)$ of the received SNR over the fading channel.Substituting (14) into (13), we obtain the average AUC over the generalized channels for the no-diversity as

$$
\bar{A}=\left.\frac{1}{u 2^{u} \Gamma(u)} \sum_{k=0}^{\infty} \frac{\Gamma(k+2 u)_{2} F_{1}(1, k+2 u ; 1+u ; 0.5)}{\Gamma(k+u) k ! 2^{(u+k)}}(-1)^{k} \phi_{\gamma}^{(k)}(s)\right|_{s=1}
$$


International Journal of Wireless \& Mobile Networks (IJWMN) Vol. 4, No. 4, August 2012

Similarly, another generic expression for the average AUC can be derived using (11) as (16).

$$
\bar{A}=1-\left.\frac{1}{\Gamma(u)} \sum_{k=0}^{\infty} \frac{\Gamma(k+2 u)_{2} F_{1}(1, k+2 u ; 1+u+k ; 0.5)}{(u+k) \Gamma(k+u) k ! 2^{(2 u+k)}}(-1)^{k} \phi_{\gamma}^{(k)}(s)\right|_{s=1}
$$

Sincek-th derivative of the MGF of the common multipath fading channelsare available, the average AUC can be readily obtained from (15) or (16). Thus,(15) and (16) provides generalizedsolutions over the myriad fading environments. For convenience, the $k$-th orderderivativesof the MGF of the end-to-end SNR of common fading channels are listed in Table 1[16]. Similarly, (15) and (16) are also applicable to the channels with composite effects (fading with shadowing). To obtain the detection performance for channels with composite effects, we just need the higher order derivative of the MGF of the effective SNR of the channel with the composite effects.

Even though we have infinite series in (15) and (16), they converge with reasonable numbers of terms with truncation error less than $.01 \%$. Table 2 shows the number of terms required for obtaining a four figure accuracy for various channels. The Table also shows that the series in (16) converges much faster than the series in (15). Herath et al. also showed the convergence for the similar Marcum-Q function in [17] with reasonable number of terms.

It is now worth mentioning that the MGF based solution also simplifies the ROC analysis. We can easily obtain the average detection probability $\bar{P}_{d}(\gamma, \lambda)$ by averaging the $P_{d}(\gamma, \lambda)((8)$ or (9)) over the PDF of channel SNR and express in terms of the higher order derivatives of the MGF of the end-to-end SNR as

$$
\begin{gathered}
\bar{P}_{d}(\gamma, \lambda)=\left.\sum_{k=0}^{\infty} \frac{(-1)^{k}}{k !} \frac{\Gamma\left(u+k, \frac{\lambda}{2}\right)}{\Gamma(u+k)} \phi_{\gamma}^{(k)}(s)\right|_{s=1} \\
\bar{P}_{d}(\gamma, \lambda)=1-\left.\sum_{k=0}^{\infty} \frac{(-1)^{k}}{k !} \frac{G\left(u+k, \frac{\lambda}{2}\right)}{\Gamma(u+k)} \phi_{\gamma}^{(k)}(s)\right|_{s=1}
\end{gathered}
$$

Thus, using Table-I, we can also easily obtain $\bar{P}_{d}(\gamma, \lambda)$ for various channel models.

\subsection{CDF Based Framework}

We were motivated to develop a CDF based framework to handle specific diversity combining scenarios. For some diversity combining cases CDF based approach has advantages over other frameworks;selection combining (SC) is such a scheme. The average AUC, based on the CDF approach can be given as (See the detail derivation in Appendix-A).

$$
\bar{A}=1-\frac{\Gamma(2 u)}{\left.2^{2 u} \Gamma(u) \Gamma(u+1)\right)} \int_{0}^{\infty} F_{\gamma}(\gamma) e_{1}^{-\gamma} F_{1}\left(2 u, u+1 ; \frac{\gamma}{2}\right) d \gamma
$$

Eqn. (19) can be solved numerically for fading channels using the CDF listed in [18, pp. 420] easily by common mathematical software packages (e.g., MATLAB, Mathematica). 
International Journal of Wireless \& Mobile Networks (IJWMN) Vol. 4, No. 4, August 2012

Table 1.MGFof SNRand its $k$-th Derivative for Several Common Stochastic Channel Models

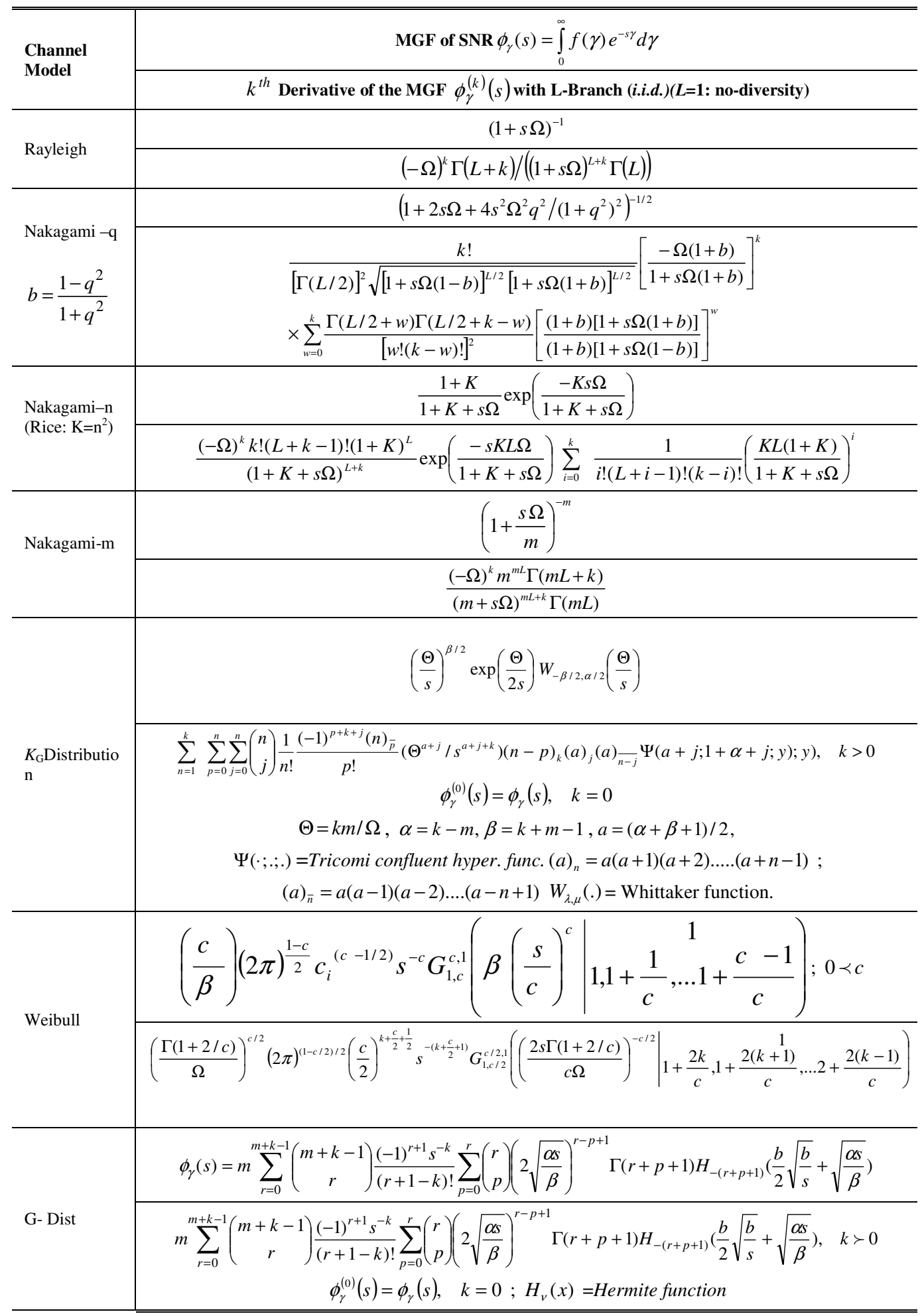

${ }^{*} L$-BRANCH for MRC/SLC 
International Journal of Wireless \& Mobile Networks (IJWMN) Vol. 4, No. 4, August 2012

Table 2.Number of Terms Required to Obtain Four Figure Accuracy (N)

\begin{tabular}{|c|c|c|c|c|c|c|}
\hline Channel & $\begin{array}{c}\mathrm{SNR}=-5 \mathrm{~dB} \\
\mathrm{u}=2\end{array}$ & $\begin{array}{c}\text { SNR }=-5 \mathrm{~dB} \\
\mathrm{u}=4\end{array}$ & 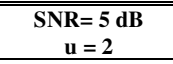 & 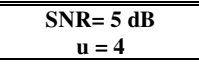 & $\begin{array}{c}\mathrm{SNR}=10 \mathrm{~dB} \\
\mathrm{u}=\mathbf{2}\end{array}$ & $\begin{array}{c}\text { SNR }=10 \mathrm{~dB} \\
\mathrm{u}=\mathbf{4}\end{array}$ \\
\hline \multirow{2}{*}{$\left|E_{N a k}\right|$} & & & & & $\mathrm{m}=4, \mathrm{~N}_{1}=49$ & $\mathrm{~m}=4, \mathrm{~N}_{1}=47$ \\
\hline & $\mathrm{m}=4, \mathrm{~N}_{2}=4$ & $\mathrm{~m}=4, \mathrm{~N}_{2}=5$ & $\mathrm{~m}=4, \mathrm{~N}_{2}=9$ & $\mathrm{~m}=4, \mathrm{~N}_{2}=11$ & $\mathrm{~m}=4, \mathrm{~N}_{2}=13$ & $\mathrm{~m}=4, \mathrm{~N}_{2}=16$ \\
\hline$\left|E_{N a k-q}\right|$ & $\begin{array}{l}q=1, N_{1}=8 \\
q=1, N_{2}=5\end{array}$ & $\begin{array}{l}q=1, N_{1}=8 \\
q=1, N_{2}=5\end{array}$ & $\begin{array}{l}\mathrm{q}=1, \mathrm{~N}_{1}=40 \\
\mathrm{q}=1, \mathrm{~N}_{2}=10\end{array}$ & $\begin{array}{l}q=1, N_{1}=38 \\
q=1, N_{2}=12\end{array}$ & $\begin{array}{c}\mathrm{q}=1, \mathrm{~N}_{1}=127 \\
\mathrm{q}=1, \mathrm{~N}_{2}=10\end{array}$ & $\begin{array}{c}\mathrm{q}=1, \mathrm{~N}_{1}=119 \\
\mathrm{q}=1, \mathrm{~N}_{2}=13\end{array}$ \\
\hline$\left|E_{\text {Rice }}\right|$ & $\begin{array}{l}K=4, N_{1}=6 \\
K=4, N_{2}=4\end{array}$ & $\begin{array}{l}K=4, N_{1}=6 \\
K=4, N_{2}=5\end{array}$ & $\begin{array}{l}\mathrm{K}=4, \mathrm{~N}_{1}=21 \\
\mathrm{~K}=4, \mathrm{~N}_{2}=11\end{array}$ & $\begin{array}{l}\mathrm{K}=4, \mathrm{~N}_{1}=21 \\
\mathrm{~K}=4, \mathrm{~N}_{2}=12\end{array}$ & $\begin{array}{l}\mathrm{K}=4, \mathrm{~N}_{1}=52 \\
\mathrm{~K}=4, \mathrm{~N}_{2}=13\end{array}$ & $\begin{array}{l}\mathrm{K}=4, \mathrm{~N}_{1}=50 \\
\mathrm{~K}=4, \mathrm{~N}_{2}=14\end{array}$ \\
\hline$\left|E_{N a k, 2(M R C)}\right|$ & $\begin{array}{c}\mathrm{m}=4, \mathrm{k}=1 \\
\mathrm{~N}_{1}=7 \\
\mathrm{~m}=4, \mathrm{k}=1 \\
\mathrm{~N}_{2}=5\end{array}$ & $\begin{array}{c}\mathrm{m}=4, \mathrm{k}=1, \mathrm{~N}_{1}= \\
7 \\
\mathrm{~m}=4, \mathrm{k}=2, \mathrm{~N}_{2}= \\
6\end{array}$ & $\begin{array}{c}\mathrm{m}=4, \mathrm{k}=1, \mathrm{~N}_{1}= \\
26 \\
\mathrm{~m}=4, \mathrm{k}=2, \mathrm{~N}_{2}= \\
12\end{array}$ & $\begin{array}{l}\mathrm{m}=4, \mathrm{k}=1, \mathrm{~N}_{1}=26 \\
\mathrm{~m}=4, \mathrm{k}=2, \mathrm{~N}_{2}=12\end{array}$ & $\begin{array}{l}\mathrm{m}=4, \mathrm{k}=1, \mathrm{~N}_{1}=66 \\
\mathrm{~m}=4, \mathrm{k}=2, \mathrm{~N}_{2}=11\end{array}$ & $\begin{array}{c}\mathrm{m}=4, \mathrm{k}=1 \\
\mathrm{~N}_{1}=65 \\
\mathrm{~m}=4, \mathrm{k}=2 \\
\mathrm{~N}_{2}=14\end{array}$ \\
\hline \multirow{2}{*}{$\left|E_{\text {Rice,2(MRC) }}\right|$} & $\mathrm{K}=2, \mathrm{~N}_{1}=7$ & $\mathrm{~K}=2, \mathrm{~N}_{1}=7$ & $\mathrm{~K}=2, \mathrm{~N}_{1}=32$ & $\mathrm{~K}=2, \mathrm{~N}_{1}=31$ & $\mathrm{~K}=2, \mathrm{~N}_{1}=88$ & $\mathrm{~K}=2, \mathrm{~N}_{1}=87$ \\
\hline & $\mathrm{K}=2, \mathrm{~N}_{2}=5$ & $\mathrm{~K}=2, \mathrm{~N}_{2}=7$ & $\mathrm{~K}=2, \mathrm{~N}_{2}=11$ & $\mathrm{~K}=2, \mathrm{~N}_{2}=13$ & $\mathrm{~K}=2, \mathrm{~N}_{2}=13$ & $\mathrm{~K}=2, \mathrm{~N}_{2}=17$ \\
\hline
\end{tabular}

* $\mathrm{N}_{1}$ represents Eq. (15); N2represents Eq. (16)

\section{DiVersity BASED ENERgY DETECTOR}

Energy detector's performance is severely limited by harsh propagation environments and becomes unreliable at low signal-to-noise ratio (SNR). Diversity combining is proposed in many works to overcome this limitation.In this Section, we have studied the diversity combining based energy detection using the frameworks developed in Section 3. Diversity based receiver receives redundantly the same information-bearing signal over multipath channels and combines multiple replicas in order to increase the overall received SNR. The combining can be done in many ways, which varies in complexity and overall performance. Common diversity reception can be done via space, frequency, time, and delay diversity[18].Diversity combining that takes place at RF is called pre-detection combining, while diversity combining that takes place at the baseband is called post-detection combining. In many cases, there is no difference in performance, at least in an ideal sense.To show the versatility of our frameworks and the efficacy of the diversity combining, we illustrate the energy detector's performance for MRC, SLC, SC,switch and stay combining (SSC) schemes for $L$ independent branchesover the Nakagami- $m$ and or Rice channels for flat fading components and over $K_{\mathrm{G}}$ distribution for the composite effects in the following sub-sections.

\subsection{Maximum Ratio Combining (MRC)}

The output SNR, $\gamma_{M R C}$ of the MRC combiner is the sum of all the SNRs on all branches, i.e., $\gamma_{M R C}=\sum_{l=1}^{L} \gamma_{l}$; where $L$ is the number of diversity branches[18]. The statistics of the decision variable of a MRC follows $\chi_{2 u}^{2}$ distribution under $H_{0}$ and $\chi_{2 u}^{2}\left(\gamma_{M R C}\right)$ under $H_{1}$ Hypothesis[9]. Therefore, the $P_{f}(\lambda)$ and the $P_{d}(\gamma, \lambda)$ at the MRC output for AWGN channels can be evaluated by (3) and (4) respectively. Thus, using the TABLE 1 for the i.i.d.L-branch, average AUC over various channels can be readily derived from (15) or (16). For instance, average AUC for i.i.d.L branches over Nakagami- $m$ and Rice channels models can be given in (20) and (21) respectively.

$$
\bar{A}(\gamma)_{N a k}^{M R C}=\frac{1}{u 2^{u} \Gamma(u)} \sum_{k=0}^{\infty} \frac{\Gamma(k+2 u)_{2} F_{1}(1, k+2 u ; 1+u ; 0.5)}{\Gamma(k+u) k ! 2^{(u+k)}} \frac{\Omega^{k} m^{m L} \Gamma(m L+k)}{(m+\Omega)^{m L+k} \Gamma(m L)}
$$




$$
\begin{aligned}
\bar{A}(\gamma)_{R i c}^{M R C}= & \frac{1}{u 2^{u} \Gamma(u)} \sum_{k=0}^{\infty} \frac{\Gamma(k+2 u)_{2} F_{1}(1, k+2 u ; 1+u ; 0.5)}{\Gamma(k+u) k ! 2^{(u+k)}} \frac{(\Omega)^{k} k !(L+k-1) !(1+K)^{L}}{(1+K+\Omega)^{L+k}} \\
& \times \exp \left(\frac{-K L \Omega}{1+K+\Omega}\right) \sum_{i=0}^{k} \frac{1}{i !(L+i-1) !(k-i) !}\left[\frac{K L(1+K)}{1+K+\Omega}\right]^{i}
\end{aligned}
$$

For general cases of i.n.d. branches, we can use the MGF of the effective SNR at the combiner as $\phi(s)_{M R C}=\prod_{i=1}^{L} \phi(s)_{i}$, where $\phi(s)_{i}$ is the MGF of the SNR of the $i$-th branch. Using the Leibniz differentiation rule [19, eq. (0.42)], we can obtain the $k$-th derivative of $\phi(s)_{M R C}$ as

$$
\phi(s)_{M R C}^{(k)}=\sum_{n_{1}=0}^{k} \sum_{n_{2}=0}^{n_{1}} \ldots \ldots \sum_{n_{L-1}=0}^{n_{L-2}}\left(\begin{array}{l}
k \\
n_{1}
\end{array}\right)\left(\begin{array}{l}
n_{1} \\
n_{2}
\end{array}\right) \ldots\left(\begin{array}{l}
n_{L-2} \\
n_{L-1}
\end{array}\right) \phi(s)_{1}^{\left(k-n_{1}\right)} \phi(s)_{2}^{\left(k-n_{2}\right)}(s) \ldots \ldots \ldots \ldots(s)_{L}^{\left(n_{L-1}\right)}
$$

Thus, using (22) in (15) or (16), average AUC can be obtained for MRC over i.n.d. branches for any fading channel.

\subsection{Square-Law Combining (SLC)}

Since the output decision is combined after the sampling, the decision variable is the sum of Li.i.d. The statistics of the decision variable of a SLC follows $\chi_{2 u}^{2}$ distribution under $H_{0}$ and $\chi_{2 L u}^{2}\left(\varepsilon_{S L C}\right)$ under $H_{1}$ Hypothesis[9]; where the non-centrality $\varepsilon_{S L C}=\sum_{l=1}^{L} \varepsilon_{l}=\sum_{l=1}^{L} 2 \gamma_{l}=2 \gamma_{S L C}$ ;hence the $P_{f}(\lambda)$ and $P_{d}(\gamma, \lambda)$ can be expressed by (3) and (4) respectively by replacing $u$ with $L u$. Thus, for the SLC scheme, average AUC can be obtained over myriad fading environments using the same derived expressions (15) or (16) just by replacing $u$ with Lu.Similar to the i.n.d. MRC, we can also obtain average AUC for the i.n.d.SLCas $\phi(s)_{S L C}=\phi(s)_{M R C}=\prod_{i=1}^{L} \phi(s)_{i}$.

\subsection{Selection Combining (SC)}

With SC, the path output from the combiner has an SNR equal to the maximum SNR of all the branches given by $\gamma_{s c}=\max \left(\gamma_{1}, \gamma_{1}, . ., \gamma_{L}\right)$. Because of the single branch considered at any given period the decision output is the i.i.d. variable and follows the $\chi_{2 u}^{2}$ for $H_{0}$ and $\chi_{2 u}^{2}\left(\varepsilon_{j}\right)$ for $H_{1}$. Since SC selects single branch at any given period, we have treated this scheme based on the developed CDF approach. For $L$ branch diversity, the CDF is given as[18]

$$
F_{\gamma_{S C}}=P\left(\max \left[\gamma_{1}, \gamma_{2}, \ldots . \gamma_{L}\right]<\gamma\right)=\prod_{l=1}^{L} P\left(\gamma_{l}<\gamma\right)=\prod_{l=1}^{L} F_{\gamma_{l}}(\gamma)
$$

where, $F_{\gamma_{l}}(\gamma)$ is the CDF of the $l$-th branch. Thus, the average AUC over the fading channels for the SC scenario can be given readily using (19) as

$$
\bar{A}^{S C}=1-\frac{\Gamma(2 u)}{\left.2^{2 u} \Gamma(u) \Gamma(u+1)\right)} \int_{0}^{\infty} F_{\gamma_{S C}}(\gamma) e^{-\gamma} F_{1}\left(2 u, u+1 ; \frac{\gamma}{2}\right) d \gamma
$$

As an illustration, average AUC can be given using the CDF [18] of link SNR overi.n.d. Nakagami- $m$ channels for the SC scheme from (24) as 


$$
\bar{A}_{N a k}^{S C}=1-\frac{\Gamma(2 u)}{2^{2 u} \Gamma(u) \Gamma(u+1)} \int_{0}^{\infty} \prod_{l=1}^{L}\left[1-\Gamma\left(\mathrm{m}_{l}, \frac{\mathrm{m}_{l}}{\Omega_{l}} \gamma\right) / \Gamma\left(\mathrm{m}_{l}\right)\right] \mathrm{e}^{-\gamma}{ }_{1} F_{1}\left(2 u, u+1 ; \frac{\gamma}{2}\right) d \gamma
$$

For i.i.d. case, (25) becomes

$$
\bar{A}_{N a k}^{S C}=1-\frac{\Gamma(2 u)}{2^{2 u} \Gamma(u) \Gamma(u+1)} \int_{0}^{\infty}\left[1-\Gamma\left(\mathrm{m}, \frac{\mathrm{m}}{\Omega} \gamma\right) / \Gamma(\mathrm{m})\right]^{\mathrm{L}} \mathrm{e}^{-\gamma}{ }_{1} F_{1}\left(2 u, u+1 ; \frac{\gamma}{2}\right) d \gamma
$$

Similarly the average AUC over the i.i.d. Rice channels can be given as

$$
\bar{A}_{R i c}^{S C}=1-\frac{\Gamma(2 u)}{2^{2 u} \Gamma(u) \Gamma(u+1)} \int_{0}^{\infty}\left[1-Q_{1}\left(\sqrt{2 K}, \sqrt{\frac{2(1+K) \gamma}{\Omega}}\right)\right]^{\mathrm{L}} \mathrm{e}^{-\gamma}{ }_{1} F_{1}\left(2 u, u+1 ; \frac{\gamma}{2}\right) d \gamma
$$

where, $Q_{1}(.,$.$) is the first order Marcum Q function. Although in[5], closed form expression is$ developed for SC scheme, it requires critical mathematical calculationswhile our CDF based approach is simple and can be solved numerically using any math package easily.Hencethis framework is more attractive compared to the one given in [5]. Moreover, the developed expression is applicable to all common fading channels. This method can also be usedsuitably for characterizing the ROC curve of average detection probability.

\subsection{Composite Channels}

Wireless channel is a complicated phenomenon and the communication is affected by not only various multipath fading but also by shadowing effects. Shadowing process is typically modelled as a lognormal distribution [20]. Practical wireless channels can be modelled as multipath fading superimposed on lognormal shadowing but due to the difficulty of analyzing, shadowing effect is commonly neglected in the literature. But in recent time, Shankar[21] showed that the generalized- $K\left(K_{\mathrm{G}}\right)$ distribution can be used to model fading and shadowing phenomena effect in mobile communication channels and this distribution makes the mathematical performance analysis much simpler to handle as compared to Lognormal-based models such as Nakagami- $m$ or the Rayleigh-Lognormal (R-L) model.To take this advantage, we considered the $K_{\mathrm{G}}$ distribution to include the composite effects in the performance analysis.

Similar to the multipath fading scenarioswe can also derive the average AUC over the composite channels (i.i.d. or i.n.d. cases) for various combining schemes. For example, the SLCover i.n.d. $K_{\mathrm{G}}$ distribution for composite effects can be given as

$$
\begin{aligned}
\bar{A}_{\text {comp }}^{S L C} & =1-\frac{1}{\Gamma(u L)} \sum_{k=0}^{\infty} \frac{\Gamma(k+2 u L)_{2} F_{1}(1, k+2 u L ; 1+u L+k ; 0.5)}{(u L+k) \Gamma(k+u L) k ! 2^{(2 u L+k)}}(-1)^{k} \\
& \times \sum_{n_{1}=0}^{k} \sum_{n_{2}=0}^{n_{1}} \ldots \ldots \sum_{n_{L-1}=0}^{n_{L-2}}\left(\begin{array}{l}
k \\
n_{1}
\end{array}\right)\left(\begin{array}{l}
n_{1} \\
n_{2}
\end{array}\right)\left(\begin{array}{l}
n_{2} \\
n_{3}
\end{array}\right) \ldots . .\left.\left.\left(\begin{array}{l}
n_{L-2} \\
n_{L-1}
\end{array}\right) \phi_{1}^{\left(k-n_{1}\right)}(s)\right|_{s=1} \phi_{2}^{\left(n_{1}-n_{2}\right)}(s)\right|_{s=1} \ldots \ldots .\left.\phi_{L}^{\left(n_{L-1}\right)}(s)\right|_{s=1}
\end{aligned}
$$

where, $\phi_{i}^{(k)}(s)$ is the $k$-th derivative of the MGF of the $i$-th channel SNR with composite effects (see Table-1, $K_{\mathrm{G}}$ distribution).For SC scheme, we follow the derived CDF approach (19). The CDF $F_{\gamma}(\gamma)$ can be given as [22] 


$$
F_{\gamma}(\gamma)=\pi \csc (\pi \alpha)\left[\frac{(\Theta \gamma)^{m}{ }_{1} F_{2}(m ; 1-\alpha, 1+m ; \Theta \gamma}{\Gamma(k) \Gamma(1-\alpha) \Gamma(1+m)}-\frac{(\Theta \gamma)^{k}{ }_{1} F_{2}(k ; 1+\alpha, 1+k ; \Theta \gamma}{\Gamma(m) \Gamma(1+\alpha) \Gamma(1+k)}\right]
$$

where, $\mathrm{pFq}(\cdot)$ is the generalized hypergeometric function[19, eq. (9.14/1)], and $\mathrm{p}, \mathrm{q}$ are integers. Thus, inserting (29) in to (24), average AUC for SC scheme can be derived over the $K_{\mathrm{G}}$ distribution easily by numerical methods.

\section{NUMERICAL RESUltS}

We have used Mathematica and MATLAB software to obtain various numerical results of the average AUC in myriad channel environments. We completed analyses in two parts; the first part includes the no-diversity and the second part includes the diversity combining scenarios for i.i.d./i.n.d.channels. But first we want to give a glimpse of AUC's appealing approachin contrast to ROC curve. We show how the AUC works as the single figure of merit and useful it could be specially when the ROC curves overlaps.Figure 1 shows the overlapping complementary ROC curves for various detectors. It is difficult to picture visually which detector gives better performance in the case of overlapping scenario. Using the average AUC expressions proposed in this article, we obtain distinct average AUC curves against the SNR, which makes the comparison much easier (Figure 2). The computed average AUC value at mean $10 \mathrm{~dB}$ for the curves (top to bottom) in Figure2 are 0.9966, 0.9898, 0.9821, 0.9663, 0.924, 0.9158. This implies that the average AUC can be a single figure of merit that characterizes the performance of an energy detector over the range of received signal SNR.

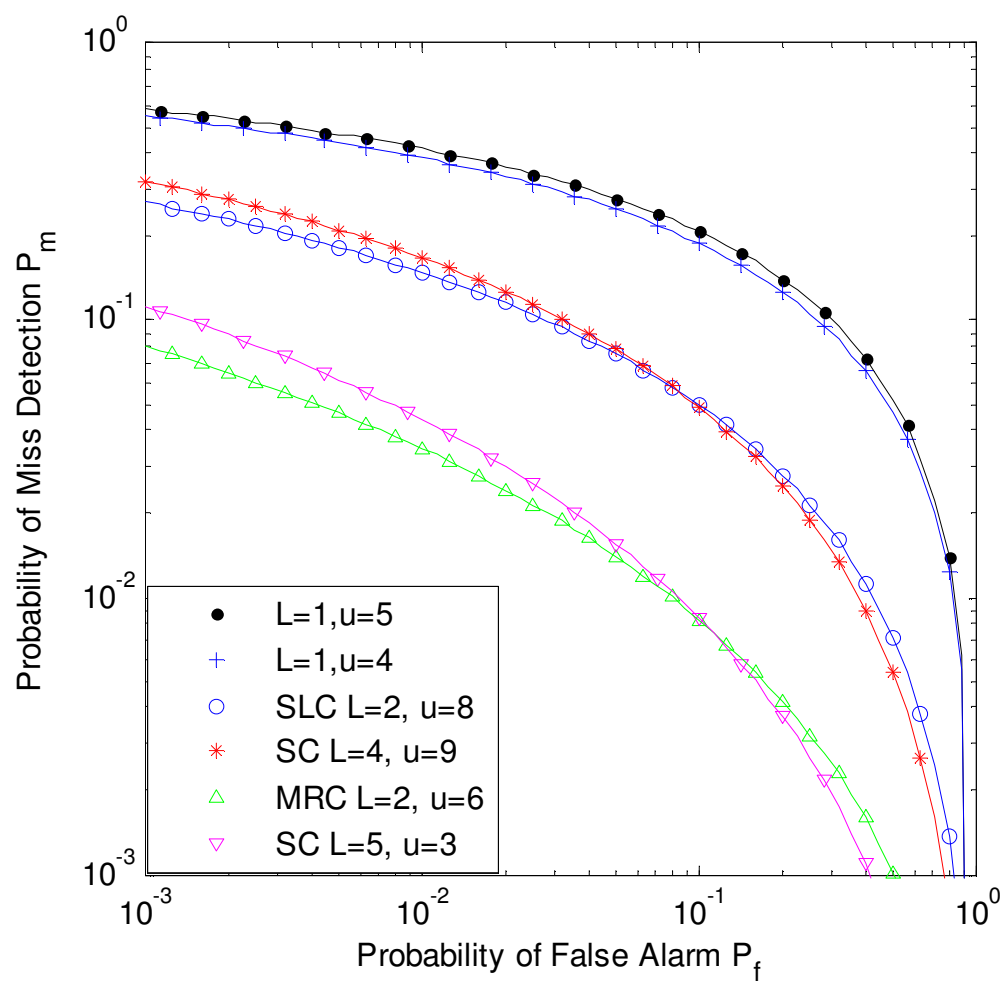

Figure 1. Complementary ROC curves of different detection configuration in Nakagami- $m$ channel, $m=2$, mean $S N R=10 \mathrm{~dB}$ 


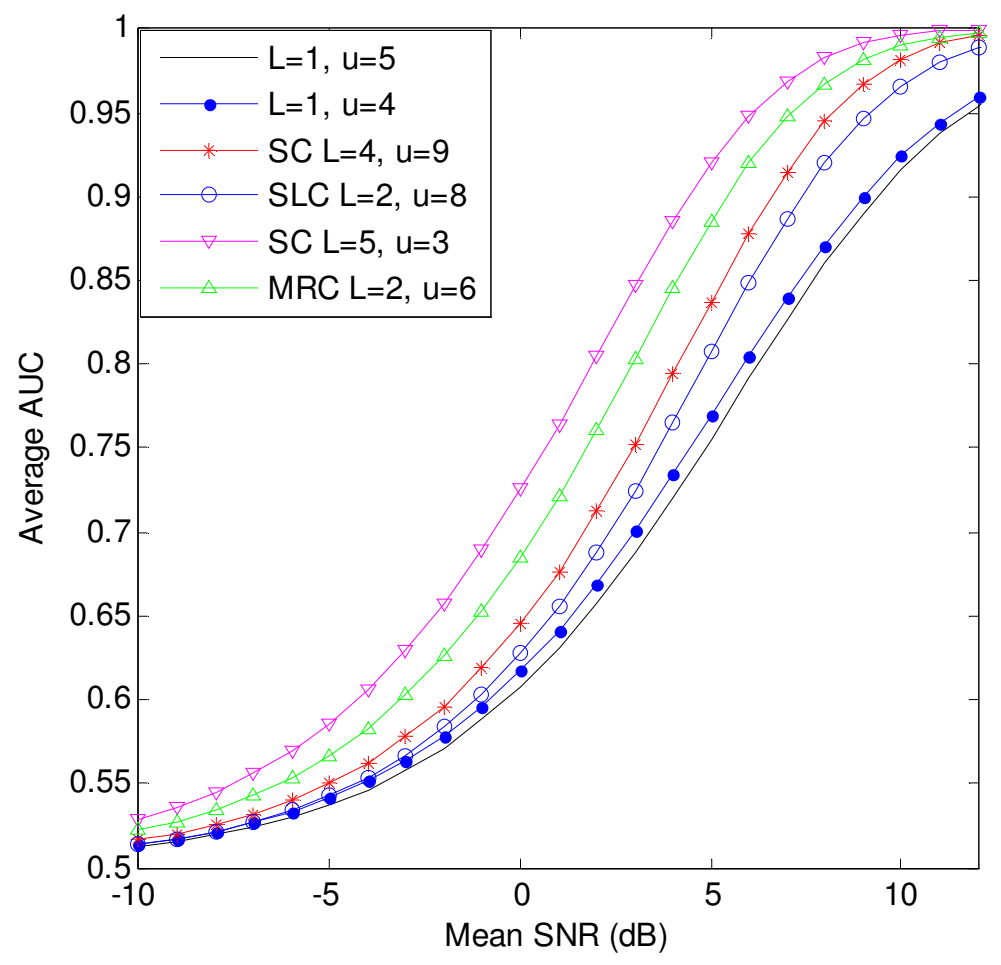

Figure 2. Average AUC vs. mean SNR for various energy detectors in Nak- $m$ channels $(m=2)$

\subsection{No-Diversity Scenarios}

In Figure 3, we present the strength of the MGF based framework by showing the performance curves in various fading channel models. The detection performance in[5] with AUC metric is analyzed only over Nakagami- $m$ fading channel and the expression is not easy to extend for generalization whereas our derived frameworks are compact and can be easily utilized for various i.i.d./i.n.d. fading channels.For illustrations we have considered the Nakagami-m,Rice, Nakagami-q and $K_{\mathrm{G}}$ distribution. Each AUC curve in Figure3shows that the performance becomes 0.5 if the detector's performance is no better than flipping a coin and increases to 1 as the performance improves. Thus, it is more meaningful to describe practical detector's performance with the AUC metric in contrast to ROC metric. Although not reported here, we noticed that at high SNR, the performance is better but for a fixed SNR, the higher sampling $(u)$ numbers do not increase the detection performance since the false alarm also increases. We also observed though not shown that the impact of Rice $K$ factor on the performance is insignificantfor curves below $0 \mathrm{~dB}$ and it affects slightly on curves over $5 \mathrm{~dB}$. When shadowing is present with the multipath fading effects, the detection performance is not as good as the case when only multipath fading is present. This can be seen by comparing the curve for the composite effects ( $K_{\mathrm{G}}$ distribution) and the Nakagami-m channel only. When $m=1$, $K_{\mathrm{G}}$ distribution becomes $K$ distribution. We also noticed that the detection performance is better over $K_{\mathrm{G}}$ distribution in comparison to only $K$ distribution (not shown here).Figure 3 also includes the curves with the half-odd integer $u$ and $m$ values, which are in sharp contrast for most existing results. 
International Journal of Wireless \& Mobile Networks (IJWMN) Vol. 4, No. 4, August 2012

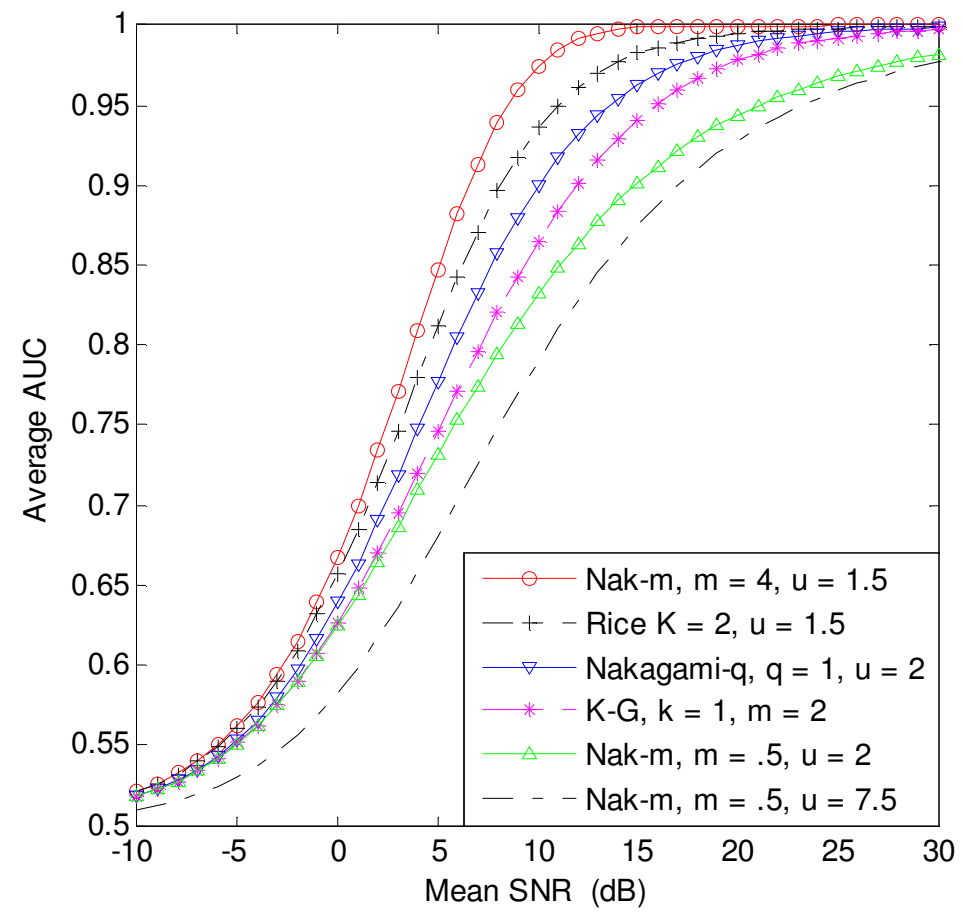

Figure 3. Average AUC vs. mean SNR for no-diversity

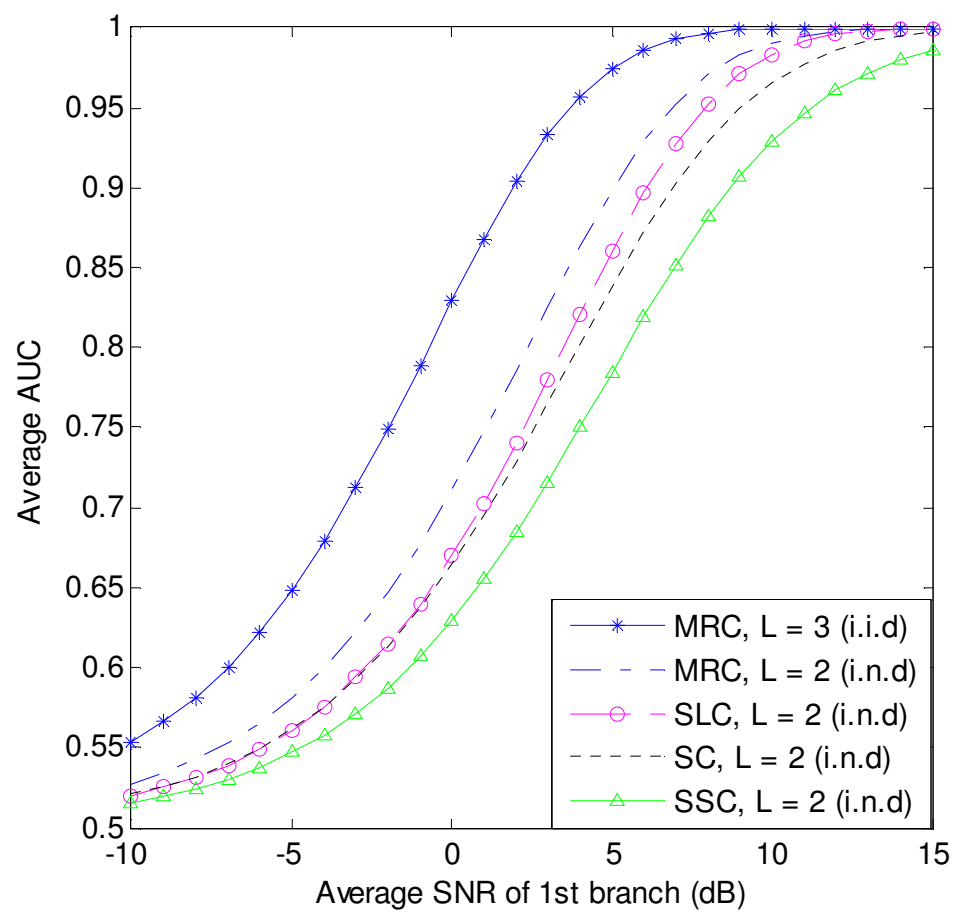

Figure 4. Average AUC vs. mean SNR with diversity combining over Nakagami- $m$ channels; $m_{1}=3, m_{2}=2 u=2, \bar{\gamma}_{1}=2 \bar{\gamma}_{2}$, where $\bar{\gamma}_{i}$ is the end-toend mean SNR for $i$-th branch. 
International Journal of Wireless \& Mobile Networks (IJWMN) Vol. 4, No. 4, August 2012

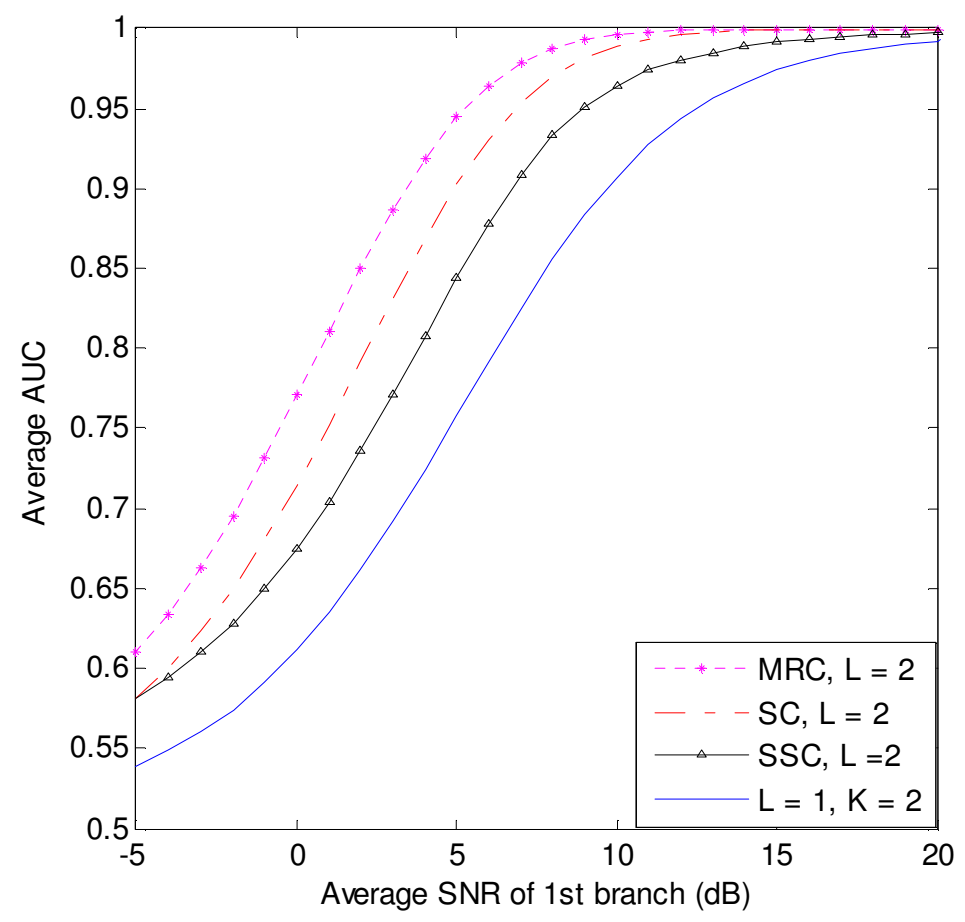

Figure 5. AUC vs. mean SNR with dual diversity combining over i.n.d. Rice fading

$$
\left(K_{1}=2, K_{2}=4, u=4.5, \bar{\gamma}_{2}=2 \bar{\gamma}_{1}\right) \text {. }
$$

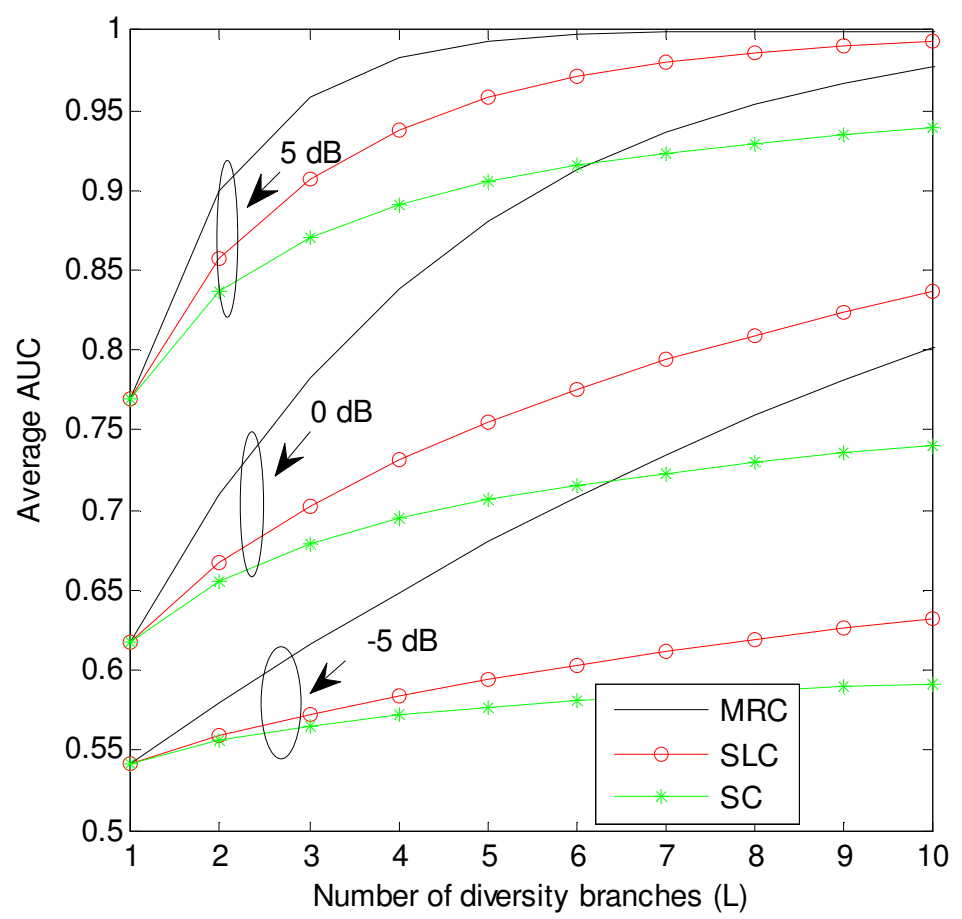

Figure 6. Average AUC vs. diversity branch $L$ over i.i.d. Nak-m channel $(u=4, m=2)$ 
International Journal of Wireless \& Mobile Networks (IJWMN) Vol. 4, No. 4, August 2012

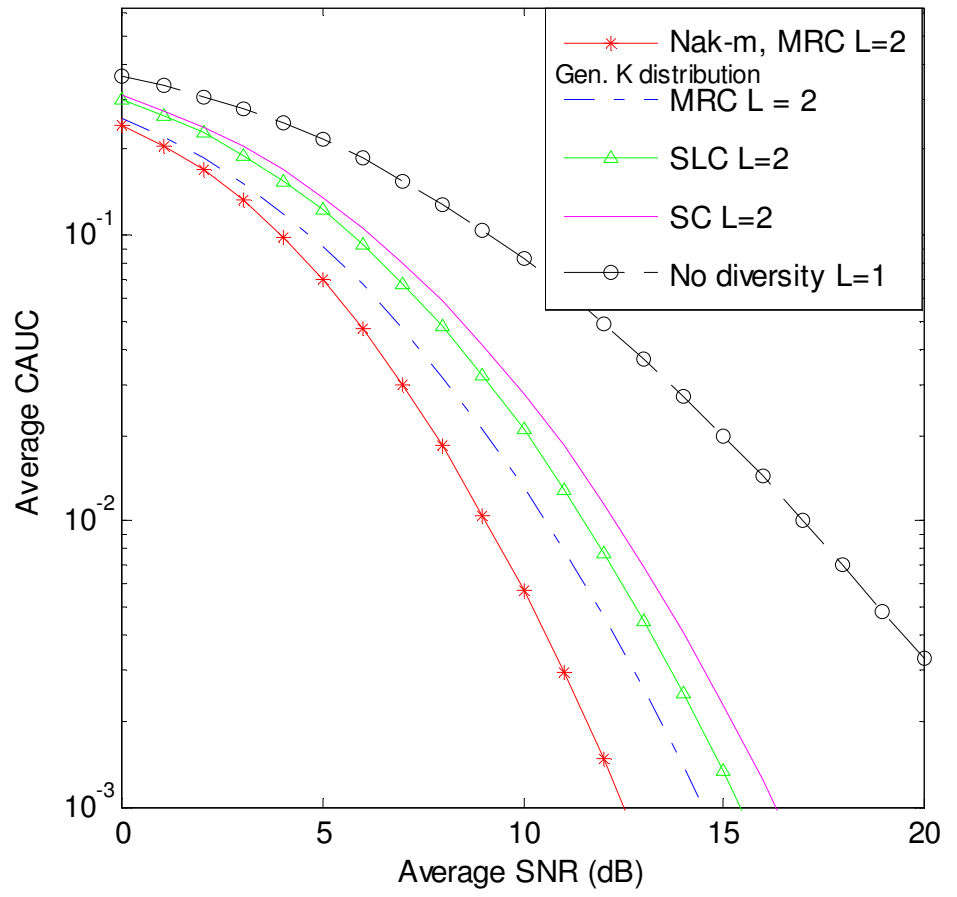

Figure 7. Average Complementary AUC (CAUC) for dual diversity reception over $K_{\mathrm{G}}$ distribution $(u=2, k=3, m=2)$

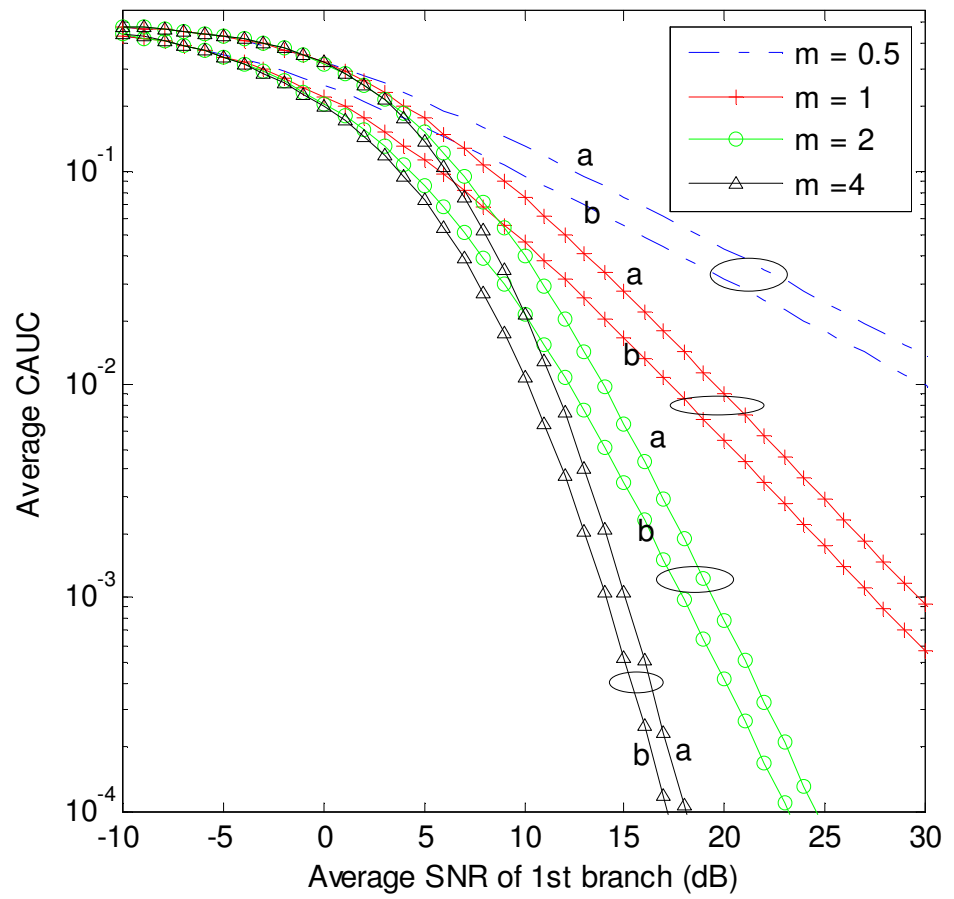

Figure 8. Comparison of (a) i.i.d. $\left(\bar{\gamma}_{2}=\bar{\gamma}_{1}\right)$ and (b) i.n.d. cases $\left(\bar{\gamma}_{2}=5 \bar{\gamma}_{1}\right)$ for SSC scheme over Nakagami- $m$ channels 
International Journal of Wireless \& Mobile Networks (IJWMN) Vol. 4, No. 4, August 2012

\subsection{Diversity Scenarios}

The developed frameworks can be applied easily for common receiver diversity combining scheme over myriad fading channels. For illustrations, we have presented results for MRC, SLC, SC and SSC schemes over Nakagami-m, Rice, and $K_{\mathrm{G}}$ distribution for i.i.d./i.n.d. cases. Mostly dual diversity combining is considered for simplicity. We used (22) either with (15) or (16) for i.n.d.MRC and SLC for demonstrating detection performance over various fading channels. For SSC, we used generalized framework (15) or (16) with the optimized threshold value[18]. For the $S C$ scheme we used the CDF based solution given in (24).

In Figure 4, we have presented detection performance for various receiver diversity combining schemes over the i.i.d. and i.n.d. Nakagami- $m$ fading channel model while in Figure5, we have produced results for various diversity combining over i.n.d. Rice fading channels. It can be noted now that our frameworks are straight forward to handle the i.n.d. cases, which were until now either intractable or intricate to solve because of the cumbersome frameworks. Figure 6 shows the impact of number of branches on the detection performance over the i.i.d. Nakagami$m$ channels. In Figure 6, it is observed that when the number of branch increases, the detection performance also increases but for higher SNR ( $>5 \mathrm{~dB})$, more than 5 branches do not make any significant difference in detection performance. In all cases (Figure4- Figure6),MRC shows the upper bounded performancebecause of the higher end-to-end SNR associated with the MRCbut in reality MRC combiner is impracticalfor simple energy detector since it is a coherent combining method; instead SLC scheme is suitable for practical implementation. In Figure7, the dual diversity reception is illustrated for various combining schemes over the i.i.d. $K_{\mathrm{G}}$ distribution for the composite effects.A comparison is also made in this plot for the MRC scheme between theNakagami- $m$ and $K_{\mathrm{G}}$ distribution. It shows that when the shadowing is present, detection performance is poor compared to one without the shadowing effect. Finally in Figure8, comparisonsare made by the complementary AUC curves between i.i.d. and i.n.d.dual diversity SSC scheme in Nakagami-m fading channels with different $m$ values. It shows that as the $m$ increases, the detection performance also improves as expected and the performance fori.n.d. shows betterfor cases when $\bar{\gamma}_{2}=n \bar{\gamma}_{1}$ (where $\bar{\gamma}_{i}$ is the end-to-end mean SNR for $i$-th branch).

In all cases, diversity combining shows better detection probability vs. no-diversity cases even at the low SNR. The composite effects are usually neglected in the research communitydue to its mathematical complexity but our frameworks are capable of handling the analytical difficulty.Our frameworks establish that they can be used with any fading environments with even half-odd integer $u$ or $m$ values (fading index of Nakagami-m channels). Thus, we have demonstrated the usefulness of the proposed frameworks and also emphasized that the AUC performance metric is a more desirable metric in contrast to ROC for describing the energy detection performance measurement.

\section{Conclusions}

We have developed two analytical approaches to characterize the AUC performance metric of energy detectors over myriad fading channel models. We then study the usefulness of the frameworks for the diversity combining techniques in i.i.d./i.n.d. wireless channels subject to multipath fading and composite effects. The results indicated that our solutions are capable ofhandling myriad fading statistics for single channels as well as for various diversity combining techniques. The results also demonstrated that at low SNR the diversity combining techniques improve the signal detection probability without any rigorous mathematics. The results could be readily used in deciding the diversity order and/or the energy threshold value required to achieve a specified false alarm rate for different operating scenarios of a cognitive radio system. 
We emphasized AUC metric to describe the energy detector's performance more meaningfully. Although AUC measurement provides simple and meaningful system performance, it is addressed only recently in the communication areas. Thus, our work adds significant values to the communication fields. The significant contribution of this work is the simplicity of our approachin analysing the energy detector's performance by involving either single fast converging series in conjunction with higher order derivative of the MGF of the channel SNR or by a CDF based single integral formula.The frameworksdo not require vigorous mathematical calculations and allow obtaining new results for several other cases for which simple solutions were not available until now. Moreover, our approach can be used with half-odd positive integer $u$ and $m$ values. To our best knowledge, use of half-odd positive integer $u$ or half-odd positive integer malone in the detection performance is not available in the literature besides our own works. Our frameworks can be easily extended for the relay based cooperative spectrum sensing and the results could readily be used to choose right parameters for energy detectors in cooperative and cognitive radio systems. Our work, thus has contributed significantly in cooperative, and cognitive communications areas.

\section{APPENDIX-A}

The average probability of detection can be given as

$$
\bar{P}_{d}=\int_{0}^{\infty} Q_{u}(\sqrt{2 \gamma}, \sqrt{\lambda}) f_{\gamma}(\gamma) d \gamma
$$

where, $f_{\gamma}(\gamma)$ is the PDF of the end-to-end link SNR. Using the integration by part and the identity[24, eq. (11)], it is straight forward to show that

$$
\bar{P}_{d}=1-\int_{0}^{\infty}\left(\frac{\lambda}{2 \gamma}\right)^{u / 2} e^{-\left(\gamma+\frac{\lambda}{2}\right)} I_{u}(\sqrt{2 \gamma \lambda}) F_{\gamma}(\gamma) d \gamma
$$

Where, $F_{\gamma}(\gamma)$ is the CDF of the link SNR and $I_{u}(\cdot)$ is the $u$-th order modified Bessel function of the first kind. Using (6) in (12), we can write:

$$
\bar{A}=-\int_{0}^{\infty} \bar{P}_{d} \frac{d P_{f}(\lambda)}{d \lambda} d \lambda
$$

Substituting (3) and (A-2) in to (A-3), we obtain

$$
\bar{A}=-\int_{0}^{\infty} \frac{\partial P_{f}}{\partial \lambda} d \lambda-\frac{1}{2^{u} \Gamma(u)} \int_{0}^{\infty} F_{\gamma}(\gamma) e^{-\gamma}(2 \gamma)^{-u / 2} I(\lambda) d \gamma
$$

where,

$$
I(\lambda)=\int_{0}^{\infty} \lambda^{\frac{3}{2} u-1} e^{-\lambda} I_{u}(\sqrt{2 \gamma \lambda}) d \lambda
$$

Substituting $u=2 n$ and using [19, eq. (6.643-2)], (A-5) can be given in terms of $u$ as (A-6). 
International Journal of Wireless \& Mobile Networks (IJWMN) Vol. 4, No. 4, August 2012

$$
I(\lambda)=\frac{\Gamma(2 u)}{\Gamma(u+1))}\left(\frac{\gamma}{2}\right)^{-1 / 2} e^{\gamma / 4} M_{\left(-\frac{3}{2} u+\frac{1}{2}\right), \frac{u}{2}}\left(\frac{\gamma}{2}\right)
$$

where $M_{k, m}(z)$ is the Whittaker function and with the identity [18, eq. (9.220-2)], (A-6) can be simplified as

$$
I(\lambda)=\frac{\Gamma(2 u)}{\Gamma(u+1))}\left(\frac{\gamma}{2}\right)_{1}^{u / 2} F_{1}\left(2 u, u+1 ; \frac{\gamma}{2}\right)
$$

where, ${ }_{1} F_{1}(\mathrm{a}, \mathrm{b} ; \mathrm{c})$ is the confluent hypergeometric function. Substituting (A-7) in (A-4), we obtain the average AUC over the fading channels as (19).

\section{REFERENCES}

[1] S. Haykin, D. Thomson, and J. Reed, "Spectrum sensing for cognitive radio," Proc. IEEE, vol. 97, no. 5, pp. 849-877, May 2009.

[2] K. Cheng and R. Prasad., Spectrum sensing in cognitive radio networks, 1st ed. New York, USA: John Wiley \& Sons, 2009, pp. 184-229.

[3] T. Fawcett, "An introduction to ROC analysis," Pattern Recognition Letter, vol. 27, no. 8, pp. 861-874, June 2006.

[4] J. Hanley and B. Mcneil, "The meaning and use of the area under a receiver operating characteristics (ROC) curve," Radiology, vol. 143, no. 1, pp. 29-36, Apr. 1982.

[5] S. Atapattu, C. Tellambura, and H. Jiang, "Analysis of area under the ROC curve of energy detection," IEEE Trans. on Wireless Commun., vol. 9, no. 3, pp. 1216-1225, March 2010.

[6] H. Urkowitz, "Energy detection of Unknown deterministic Signals," Proc. IEEE, vol. 55, no. 4, pp. 523-531, April 1967.

[7] F. Digham, M. Alouini, and M. Simon, "On the energy detection of unknown signals over fading channels," IEEE Transaction on Communications, vol. 55, no. 1, pp. 21-24, Jan 2007.

[8] K. Hemachandra and N. Beaulieu, "Novel analysis for performance evaluation of energy detection of unknown deterministic signals using dual diversity," in Proc. IEEE Veh. Technol. Conf., 2011, pp. 1-5.

[9] S.P. Herath, N. Rajatheva, and C. Tellambura, "Unified Approach for Energy Detection of Unknown Deterministic Signal in Cognitive Radio Over Fading Channels," in Proc. IEEE ICC Workshops, 2009, pp. 1-5.

[10] B. Modi, A. Annamalai, O. Olabiyi, and C. Palat, "Ergodic capacity analyses of cooperative amplify and forward relay networks over Rice and Nakagami fading channels," International Journal of Wireless and Mobile Networks, vol. 4, no. 1, pp. 97-116, Feb. 2012.

[11] S. Alam, O. Odejide, O. Olabiyi, and A. Annamalai, "Further Results on Area under the ROC Curve of Energy Detectors over Generalized Fading Channels," in Proc. 34th IEEE Sarnoff Symp., NJ, 2011.

[12] K. Letaief and W. Zhang, "Cooperative communications for cognitive radio networks," Proc. IEEE, vol. 97, no. 5, pp. 878-893, May 2009.

[13] F. Digham, M. Alouini, and M. Simon, "On the energy detection of unknown signals over fading channels," in Proc. IEEE Int. Conf. Commun., vol. 5, 2003, pp. 3575-3579.

[14] A. Liu, E. Schisterman, and C. Wu, "Nonparametric estimation and hypothesis testing on the 
partial area under receiver operating," Commun. Statistics - Theory and Methods, vol. 34, no. 9, pp. 2077-2088, Oct. 2005.

[15] A. Annamalai, C. Tellambura, and J. Matyjas, "A new twist on the generalized Marcum Qfunction QM (a, b) with fractional-order $\mathrm{M}$ and its applications," in Proc. IEEE Con. Commun. and Networking. Conf., 2009, pp. 1-5.

[16] A. Annamalai and C Tellambura, "An MGF-derivative based unified analysis of incoherent diversity reception of M-ary orthogonal signals over fading channels,"," in Proc. 54th IEEE Veh. Technol. Conf., 2001, pp. 2404 - 2408.

[17] S.P. Herath, N. Rajatheva, and C. Tellambura, "On the energy detection of unknown deterministic signal over Nakagami channels with selection combining," in IEEE CCECE '09, 2009, pp. 745-749.

[18] M. Simon and M. Alouini, Digital Communication over Fading Channels, 2nd ed. New York, USA: Wiley, 2005.

[19] I. Gradshteyn and I. Ryzhik, Table of integrals, series and products. San Diego, CA, USA: Academic, 2007.

[20] G. L. Stuber, Principles of Mobile Communication, 2nd ed. Norwell, MA: Kluwer Academic, 2001.

[21] P. M. Shankar, "Error rates in generalized shadowed fading channels," Wireless Personal Communications, vol. 28, no. 4, pp. 233-238, Feb. 2004.

[22] P. Bithas, N. Sagias, P. Mathiopoulos, G. Karagiannidis, and A. Rontogiannis, "On the performance analysis of digital communications over generalized- $K$ fading channels," IEEE Commun. Lett., vol. 10, no. 5, pp. 353-355, May 2006.

[23] S. P. Herath and N. Rajatheva, "Analysis of equal gain combining in energy detection for cognitive radio over Nakagami channels," in IEEE GLOBECOM , 2008, pp. 1-5.

[24] A. Annamalai and C. Tellambura, "A simple exponential integral representation of the generalized Marcum Q-function QM (a, b) for real-order M with applications," in Proc. 54th IEEE MILCOM, 2008, pp. 1-7.

Shumon Alam received MS degree in Electrical Engineering from Prairie View A\&M University (PVAMU), Texas in 2004 and in Telecommunications from Oklahoma State University in 2000. He earned his BS degree in Physics from University of Dhaka. He is currently working towards the Ph.D. degree in Electrical Engineering at Prairie View A\&M University. He has over 7 years of research experience in Telecommunications at the Center of Excellence for Communication Systems Technology Research (CECSTR), PVAMU. His current research interests include cooperative spectrum sensing, cognitive radio network, signal processing, network architecture, and cross layer design for wireless communications.

Oluwatobi O. Olabiyi received the B.Sc. degree in Electronic \& Electrical Engineering from ObafemiAwolowo University, Ile-Ife, and the M.S. degree in Electrical Engineering from Prairie View A\&M University, Texas. He has coauthored more than 25 publications, presented at international conferences and published in highly referred journals. He was the recipient of the Roy G. Perry College of Engineering Outstanding Masters Student of the Year Award (2011) and the National Society of Black Engineer's Golden Torch Award for Graduate Student of Year (2012). He is presently a PhD candidate at Prairie View A\&M

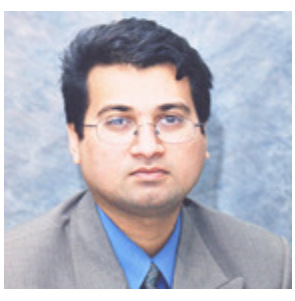
University. His research interests are in wireless communications including, dynamic spectrum access, MIMO and cooperative communications, statistical signal processing, compressive sensing, machine learning and optimization. 
Olusegun O. Odejidereceived the B.S. degree in Electrical and Electronic engineering from University of Ibadan, Ibadan, Nigeria in 1997. He received the M.S. degree in Computer and Information Systems Engineering from Tennessee State University in 2004 and Ph.D degree in Electrical Engineering from Prairie View A\&M University, Prairie View, Texas in 2009. His research interests are in the areas of Signal/Image/Video Processing and Communication Systems.

Dr. Annamalai is presently the Director of Center of Excellence for Communication Systems Technology Research, a Texas A\&M Board of Regents approved University Research Center at the Prairie View A\&M University, and a tenured faculty member in the Department of Electrical and Computer Engineering. He has over 16 years of research/teaching experience in wireless communications at Motorola, University of Victoria, Air Force Research Laboratory, Virginia Tech and PVAMU with approximately 200 peerreviewed publications and 5 book chapters. Dr. Annamalai has been honored by

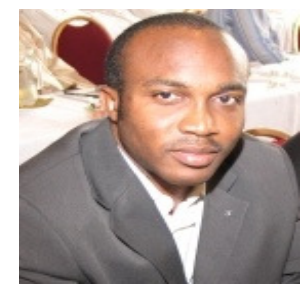
his colleagues on numerous occasions for his excellence in research including winning the 2011 Roy G. Perry College of Engineering Outstanding Faculty (Research) Award, IEEE Leon Kirchmayer Prize Paper award, ASEE/AFOSR Summer Faculty Fellowships, NSERC Doctoral Prize, CAGS/UMI Distinguished Doctoral Dissertation Award, IEEE VTS/Motorola Daniel E. Noble Fellowship, among others. He had served on the Editorial Boards of four IEEE journals/transactions in the last 12 years, and has helped to organize a few major IEEE conferences on wireless communications including serving in the capacity of Technical Program Chair of the 2002 IEEE Vehicular Technology Conference in Vancouver, Canada. His current research interests include cooperative spectrum sensing, compressive sensing, cross-layer design for scalable multimedia transmission and cooperative wireless communications. 